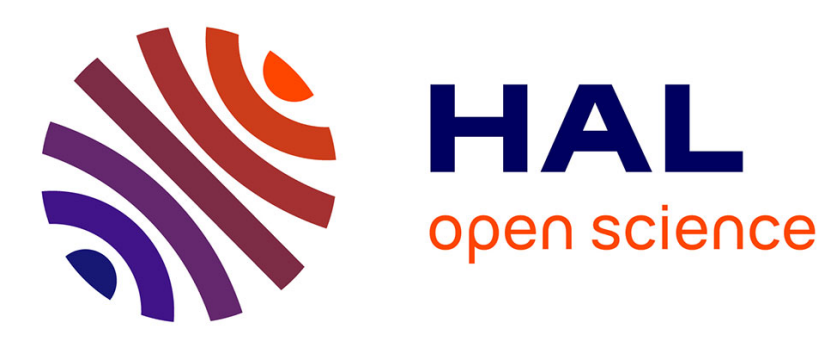

\title{
Reciprocity gap method
}

Stéphane Andrieux, Hui Duong Bui, Andrei Constantinescu

\section{To cite this version:}

Stéphane Andrieux, Hui Duong Bui, Andrei Constantinescu. Reciprocity gap method. Michel Grédiac; François Hild; André Pineau. Full-Field Measurements and Identification in Solid Mechanics, WileyVCH, pp.368-378, 2012, 978-1-84821-294-7. 10.1002/9781118578469.ch13 . hal-00762187

\section{HAL Id: hal-00762187 \\ https://hal-polytechnique.archives-ouvertes.fr/hal-00762187}

Submitted on 19 Feb 2020

HAL is a multi-disciplinary open access archive for the deposit and dissemination of scientific research documents, whether they are published or not. The documents may come from teaching and research institutions in France or abroad, or from public or private research centers.
L'archive ouverte pluridisciplinaire HAL, est destinée au dépôt et à la diffusion de documents scientifiques de niveau recherche, publiés ou non, émanant des établissements d'enseignement et de recherche français ou étrangers, des laboratoires publics ou privés.

\section{(c)(1)}

Distributed under a Creative Commons Attribution| 4.0 International License 


\section{Reciprocity Gap Method}

\subsection{Introduction}

The class of problems discussed in this chapter concerns the identification of sources, cracks, boundary conditions or physical parameters on the inner surfaces of a body for which we possess overspecified data on the outer surface of the body.

The direct problem is usually defined as the determination of a solution inside the body from knowledge of its geometry, material data and boundary conditions. The so-called "primal formulation" describes the physical phenomenon and involves the "natural boundary conditions" of the problem, which ensures the existence and uniqueness of the solution. For a given mathematical operator representing the physical phenomenon under scrutiny, for example electrical or thermal diffusion or linear elasticity, overspecified data denote pairs of fields, typically representing Neumann, Dirichlet or Robin boundary conditions when the Laplace operator is under examination. Let us remark that for a well-defined direct problem, only one of the specified fields is necessary to ensure the existence of the solution of the direct problem.

In the case of an elastic problem, knowing both tractions and displacements at the surface for a given problem setting constitutes an overspecified data pair. A similar pattern for heat diffusion would be the simultaneous knowledge of the temperature field and the heat flux on the boundary.

The classic approach to solve this type of problem is to define the unknowns (cracks, inclusions, etc.) as a finite set of parameters, denoted by a vector $\boldsymbol{x}$. The 
overspecified data will be split into an "input" and an "output". The direct problem setting will provide now a solution $u(\boldsymbol{x})$ using the input data and the "output" can finally be compared with the additional output data. The two output data sets are compared in almost all cases using a least squares cost functional expressed as $M C\left(u-u^{m}\right)$, where $u$ and $u^{m}$ are the computed and the measured output data, respectively. The identification method becomes therefore a minimization algorithm:

$$
\underset{\boldsymbol{x}}{\arg \min } M C\left[u(\boldsymbol{x})-u^{m}\right]
$$

The method is straightforward and can easily be implemented if a solver of the direct problem is available.

This method requires numerous solutions to the direct problem and does not usually enable the formulation of identifiability results, that is specifying whether or not the available data are sufficient to uniquely identify the sought unknowns.

In the particular case where the data are overspecified on the complete outer surface of the solid, another approach, called reciprocity gap, can be used. This allows us to:

- obtain without solving direct problems an infinite quantity of information concerning the unknown elements;

- identify explicitly the elements in certain particular configurations.

The reciprocity gap method relies on a simple idea, generally denoted as reciprocity, which underlines the symmetry of the operator describing the underlying physics (steady-state diffusion, linear elasticity, etc.). Depending on the area of application, reciprocity takes different names according to the physical matter: Maxwell-Betti reciprocity in elastostatics, Rayleigh reciprocity in harmonic elastodynamics, etc.

This property states that for a solid subjected to two different "loads" $C_{1}$ and $C_{2}$ and presenting the different responses $R_{1}$ and $R_{2}$, respectively, the "work" of crossed solicitations will be equal. More specifically, the "work" defined as the loading $C_{1}$ in response $R_{2}$ equals that of $C_{2}$ in $R_{1}$. This fundamental property defines self-adjoined operators in mathematical theories and leads to symmetric "stiffness" matrices in the finite element methods.

The idea behind the reciprocity gap method is to analyze the gap in reciprocity between the actual field in the real solid that provided the measurements and a fictitious solution field in a fictitious solid in the absence of the unknown elements (cracks, inclusions, sources, etc.). When comparing the two fields, the property of reciprocity is not verified; however, the scalar value of this difference provides access to the "difference" between the real domain and the fictitious solid in the absence 
of the unknown elements. This difference will further enable the identification of the unknown elements.

As a fictitious solid, we can take the homogeneous solid that can be embedded in an infinite domain, and as such we gain access to an infinite class of closed-form solutions of the direct problem for the fictitious domain. The reciprocity gap provides information about the unknowns from a straightforward integral computation on the boundary.

In several cases, the acquired information can lead to theoretical identifiability results and provide a convenient reconstruction method based on closed-form expressions for the unknowns. One such case is the identification of flat (planar) cracks in electrostatics.

In this chapter, we present three simple examples of identification using the reciprocity gap: (1) identification of planar cracks in steady-state diffusion (thermal or electrical conduction), (2) thermal sources and (3) planar cracks in linear thermoelasticity using only displacement fields for the identification.

\subsection{The reciprocity gap method}

The Maxwell-Betti reciprocity theorem in elasticity is equivalent to the symmetry property of the bilinear form $a(\boldsymbol{u}, \boldsymbol{v})$, describing the weak formulation of the direct problem. Let us consider the balance of linear momentum of an elastic body $\Omega$, with a boundary $\partial \Omega$ and unit outward normal $\boldsymbol{n}$ :

$$
\begin{aligned}
& a(\boldsymbol{u}, \boldsymbol{v})=a(\boldsymbol{v}, \boldsymbol{u}) \quad \forall(\boldsymbol{u}, \boldsymbol{v}) \in \mathcal{V}^{2} \\
& a(\boldsymbol{u}, \boldsymbol{v})=\int_{\Omega} \varepsilon[\boldsymbol{u}]: \boldsymbol{A}: \varepsilon[\boldsymbol{v}] d \Omega
\end{aligned}
$$

where $\boldsymbol{A}$ denotes the fourth-order elastic stiffness tensor and $\mathcal{V}$ denotes the set of displacement fields with finite deformation energy.

Using a similar pattern, we can access a Maxwell-Betti-type reciprocity property for all operators describing a physical setting, provided the underlying operator has the previous symmetry. The following list provides a series of examples of symmetric bilinear operators and the corresponding operators of natural boundary conditions $\sigma$ :

- harmonic elastodynamics at fixed frequency:

$$
\begin{aligned}
a(\boldsymbol{u}, \boldsymbol{v}) & =\int_{\Omega} \varepsilon[\boldsymbol{u}]: \boldsymbol{A}: \varepsilon[\boldsymbol{v}] d \Omega-\omega^{2} \int_{\Omega} \rho \boldsymbol{u} \cdot \boldsymbol{v} d \Omega \\
\sigma[\boldsymbol{u}] \cdot \boldsymbol{n} & =(\boldsymbol{A}: \varepsilon[\boldsymbol{u}]) \cdot \boldsymbol{n}
\end{aligned}
$$


where $\boldsymbol{u}, \boldsymbol{v}$ are the real and virtual displacement fields, $\varepsilon$ is the small strain operator and $\sigma$ is the stress operator.

- steady-state diffusion (electric conduction heat problem):

$$
\begin{aligned}
a(u, v) & =\int_{\Omega} \nabla u \cdot \boldsymbol{k} \cdot \nabla v d \Omega \\
\sigma[u] \cdot \boldsymbol{n} & =(\boldsymbol{k} \cdot \nabla u) \cdot \boldsymbol{n}
\end{aligned}
$$

where $u, v$ are the real and virtual potential temperature or electrical potential fields, respectively, and $\sigma$ is the operator describing the boundary flux.

- harmonic acoustics (Helmholtz equation):

$$
\begin{aligned}
a(u, v) & =\int_{\Omega} \nabla u \cdot \boldsymbol{k} \cdot \nabla v d \Omega-\omega^{2} \int_{\Omega} u \cdot v d \Omega \\
\sigma[u] \cdot \boldsymbol{n} & =(\boldsymbol{k} \cdot \nabla u) \cdot \boldsymbol{n}
\end{aligned}
$$

where $u, v$ are the real and virtual acoustic potential fields, and $\sigma$ is the acoustic flux operator.

The general setting is therefore a problem that admits a weak formulation of the type $a(u, v)=l(v)$, where $l(v)$ is the linear application of the work of surface forces. The work $l(v)$ is associated with the natural boundary conditions of the problem under consideration and $a$ is a symmetric bilinear application.

The reciprocity property states that two linear applications $l^{1}$ and $l^{2}$, expressing the work of external forces producing the solutions $u^{1}$ and $u^{2}$, respectively, of the underlying problems:

$$
a\left(u^{i}, v\right)=l^{i}(v) \quad \forall v \in \mathcal{V} \quad i=1,2
$$

will equate the crossed work of the corresponding solutions:

$$
l^{1}\left(u^{2}\right)=l^{2}\left(u^{1}\right)
$$

The boundary integrals involve the operator $\sigma$ of the natural boundary conditions associated with the bilinear application $a$, defined in the next equation:

$$
\forall u \in \mathcal{V}: \quad a(u, v)=\int_{\partial \Omega}(\sigma[u] \cdot \boldsymbol{n}) \cdot v d s \quad \forall v \in \mathcal{V}
$$

The precise definition of $\sigma$ for a series of physical models was presented in the previous list. 
The identification problems discussed in this chapter can be expressed using this general framework under the following form:

The measured experiment or the applied load leads to a field u verifying:

$$
a_{0}(u, w)+a_{1}(u, w)=l(w)+\int_{\partial \Omega_{\mathrm{ext}}} f \cdot w d s \quad \forall w \in V
$$

In this expression, the linear application $l$ (denoting sources or boundary conditions on interior boundaries) and the bilinear application $a_{1}$ (denoting material characteristics, inclusions, etc.) are unknown. We possess complete field measurements of $u=U$ on the complete boundary of the domain.

The inverse problem seeks to determine $l$ and $a_{1}$ from the overspecified boundary $\operatorname{data}(U, f)$.

\subsubsection{Definition of the reciprocity gap}

The reciprocity gap is a linear application defined over the space of potentials with finite energy by the following expression:

$$
\mathcal{R}=\int_{\partial \Omega_{\mathrm{ext}}}\left(f \cdot v-U \sigma_{0}[v] \cdot \boldsymbol{n}\right) d s
$$

This linear application is computed by integration on the external boundary of the domain and involves only known quantities. Hence, for its computation, we do not need to solve any direct problem.

The interest of this definition lies within the fundamental property of the reciprocity gap, which involves as fictitious fields $v$ of $\mathcal{V}$ "in balance" under the known bilinear form $A_{0}$ with surface fluxes and tractions on the outside boundary. Let us further denote this space by $\mathcal{V}_{A_{0}}^{e q}$. A complete definition is given by:

$$
\mathcal{V}_{a_{0}}^{e q}=\left\{v \in \mathcal{V} \mid a_{0}(v, w)=\int_{\partial \Omega_{\mathrm{ext}}}\left(\sigma_{0}[v] \cdot \boldsymbol{n}\right) \cdot w d s \quad \forall w \in \mathcal{V}\right\}
$$

\subsubsection{Fundamental property of the reciprocity gap}

For all potential fields, the reciprocity gap can also be expressed using the following expression:

$$
\mathcal{R}(v)=\ell(v)-a_{1}(u, v) \quad \forall v \in \mathcal{V}_{a_{0}}^{e q}
$$


The proof of this property is straightforward. Indeed, taking as a test field $w=u$ in the characterization of $v$, and $w=v$ in the equilibrium condition of $u$, we obtain only:

$$
a_{0}(v, u)=\int_{\partial \Omega_{\mathrm{ext}}}\left(\sigma_{0}[v] \cdot \boldsymbol{n}\right) \cdot u d s
$$

and

$$
a_{0}(u, v)+a_{1}(u, v)=\ell(v)+\int_{\partial \Omega_{\mathrm{ext}}} f \cdot v d s
$$

The sought equality follows from the difference of the previous equation, simple algebra and the symmetry of the bilinear application $a_{0}$, which is the essential property here.

For each equilibrated potential field $v$, we can obtain, by computing the reciprocity $\mathcal{R}(v)$ directly from its definition, that is a boundary integration, valuable information about the main unknowns of the problems $\ell$ and $a_{1}$. The technique of the reciprocity gap method is reduced essentially to the wise choice of the fictitious potentials $v$. This technique leads to either theoretical identification results or constructive partial or complete reconstruction results. The theoretical identification results answer the following question: does a unique pair $\left(\ell, a_{1}\right)$ exist? In other words, does there exist a single set of sources, cracks, inclusions for which the response of the solid coincides with the measured boundary data? The reconstruction techniques provide methodologies for completely or partially characterizing inclusions, cracks, sources, etc., contained in the solid.

The chapter next presents a series of examples of such results. Note that no direct problem solving is required to apply the reciprocity gap method if analytical expressions of the auxiliary fields $v$ are available. The calculations are limited to integrations on the surface of the body under scrutiny. This specificity of the method gives rise to an extremely fast and efficient identification technique. However, we should take into consideration its two major limitations: (1) the need for data over the entire boundary of the solid and (2) the lack of a systematic method for choosing the fictitious potential fields $v$.

Some proposals for reducing both limitations have been made, but they are beyond the scope of this chapter.

\subsection{Identification of cracks in electrostatics}

In the case of flat planar cracks, it is possible to formulate closed-form expressions of the normal of the plane containing one or more cracks, the complete position of the 
plane and even the complete extension of the crack in this plane. Let us now analyze the case of the steady-state heat conduction problem.

In thermal balance, if the heat flux over the boundary is known to be equal to $f$, the partial differential equations of the underlying problem are described for a thermal conductivity $k$ and a temperature field $T$ as:

$$
\operatorname{div}(k \nabla T)=0 \quad \text { in } \Omega, \quad k \nabla T \cdot \boldsymbol{n}=f \quad \text { on } \partial \Omega_{\mathrm{ext}}
$$

If the body under discussion, $\Omega$, contains a crack $\Gamma$, its boundary $\partial \Omega$ is defined by the external surface $\partial \Omega_{\mathrm{ext}}$ and the crack lips $\Gamma_{j}, j=1, N$ (see [13.1]).

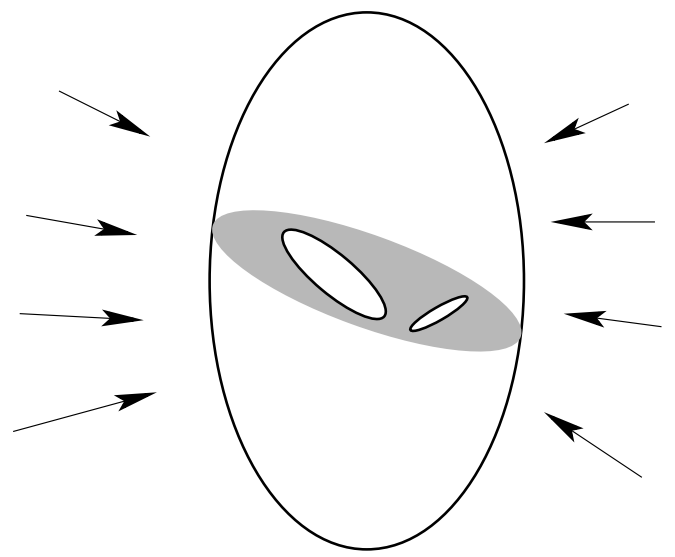

Figure 13.1. Flat planar cracks in the interior of a body

Let us assume that on the crack lips $\Gamma_{j}, j=1, N$, the boundary conditions are defined as a nonlinear contact resistivity, that is a relation representing the normal flux with the temperature jump over the lips: $\llbracket T \rrbracket$.

$$
k \nabla T \cdot \boldsymbol{N}_{j}=r_{j}(\llbracket T \rrbracket) \text { on } \Gamma_{j}, \quad \forall j=1, \text { etc., } N
$$

Let us finally assume that the temperature is measured on the outer surface of the body and the field $T^{m}$ is known.

Under these conditions, the reciprocity gap is defined as:

$$
\mathcal{R}(v)=\int_{\Omega_{\mathrm{ext}}}\left[f \cdot v-(k \nabla v \cdot \boldsymbol{n}) \cdot T^{m}\right] d s
$$


A series of straightforward computations conclude that the fundamental property of the reciprocity gap is given by:

$$
\mathcal{R}(v)=\sum_{j=1, N} \nabla v \cdot \boldsymbol{N}_{j} \llbracket T \rrbracket d s \quad \forall v \text { such that } \operatorname{div} k \nabla v=0 \quad \text { in } \quad \Omega
$$

\subsubsection{Identification formulas for the plane of the crack(s)}

Let us suppose that the cracks are situated within the same plane $\Pi$ of unit normal $\boldsymbol{N}$. The choice of linear fictitious auxiliary functions $v$, more precisely coordinate projections of the current point $\boldsymbol{x}$ :

$$
x_{i}(\boldsymbol{x})=x_{i} \quad i=1,2,3
$$

leads to the following formulas defining the unit normal of the plane and the mean value of the temperature jump over the crack:

$$
\boldsymbol{N}=\frac{\boldsymbol{L}}{\|\boldsymbol{L}\|} \quad \boldsymbol{L}=\mathcal{R}\left(x_{i}\right) \boldsymbol{e}_{i} \quad i=1,3
$$

and

$$
\left\|\sum_{j=1}^{N} e_{j} \int_{\Gamma_{i}} \llbracket T \rrbracket d s\right\|=\frac{1}{k}\|L\|,
$$

respectively.

Similarly, we can completely determine the plane $\Pi$ using quadratic fields. In this case, it is important to be aware of the fact that not all quadratic fields are harmonic and, therefore, do not satisfy the balance conditions over a homogeneous body, that is an area without cracks. However, by effecting a change of reference frame $x_{k} k=$ $1,2,3$ with coordinate $x_{3}$ directed along the normal $\boldsymbol{N}$ that has just been determined, the equation for plane $\Pi$ is given by $x_{3}+C=0$. Constant $C$ is obtained by using the auxiliary field:

$$
v(\boldsymbol{x})=x_{2}^{2}-x_{3}^{2}
$$

As the normal to the crack plane is oriented toward $x_{3}$, we obtain:

$$
\nabla v \cdot N=-2 x_{3}
$$

The integration of reciprocity for the heat equation [13.22] over the plane of the crack defined by $x_{3}+C=0$, for the particular field $v$ defined previously, leads to the following value of the constant:

$$
C=\frac{1}{2\|\boldsymbol{L}\|} \mathcal{R}\left(x_{2}^{2}-x_{3}^{2}\right)
$$


These formulas show that a single measurement identifies the plane including the cracks and that its determination requires only four simple calculations:

(i-iii) for $\mathrm{i}=1,2,3$ :

$$
\mathcal{R}\left(x_{i}\right)=\int_{\Omega_{\mathrm{ext}}}\left[f x_{i}-k n_{i} T^{m}\right] d s
$$

(iv)

$$
\mathcal{R}\left(x_{2}^{2}-x_{3}^{2}\right)=\int_{\Omega_{\mathrm{ext}}}\left[f\left(x_{2}^{2}-x_{3}^{2}\right)-k\left(x_{2} n_{2}-x_{3} n_{3}\right) T^{m}\right] d s
$$

An illustration of an indentified crack plane is shown in Figure 13.3. It is interesting to note that better accuracy for the plane position is obtained in a finite element computation when it is quadratic in the calculation of the temperature $T$ and the auxiliary fields. The error observed for linear elements derives directly from the approximation error of quadratic fields with such elements.

The identifiability condition derives from the previous arguments:

$$
\int_{\Gamma} \llbracket T \rrbracket d s \neq 0
$$

This condition is interpreted simply by the requirement that the stress applied to a solid generally "excites" the cracks. The cracks are detectable only if they are illuminated by a temperature discontinuity (because the source absence imposes the continuity of heat flow). If the loading does not lead to these discontinuities, the test is not relevant. Figure 13.2 shows such a situation.

\subsubsection{Complete identification of cracks}

Once the crack plane is completely determined, it is possible to choose further auxiliary fields to completely identify the extension of the crack, and more precisely to determine their position and shape in the plane. The shape in the plane will be reconstructed from the Fourier decomposition of the temperature jump in plane $\Pi$. The reconstruction is based on a mathematical result that ensures that the support of the cracks coincides with the support of the temperature discontinuities. In other words, it is physically impossible for the temperature discontinuity to vanish on a measureable part of the finite field occupied by the cracks (see Figure 13.4). 

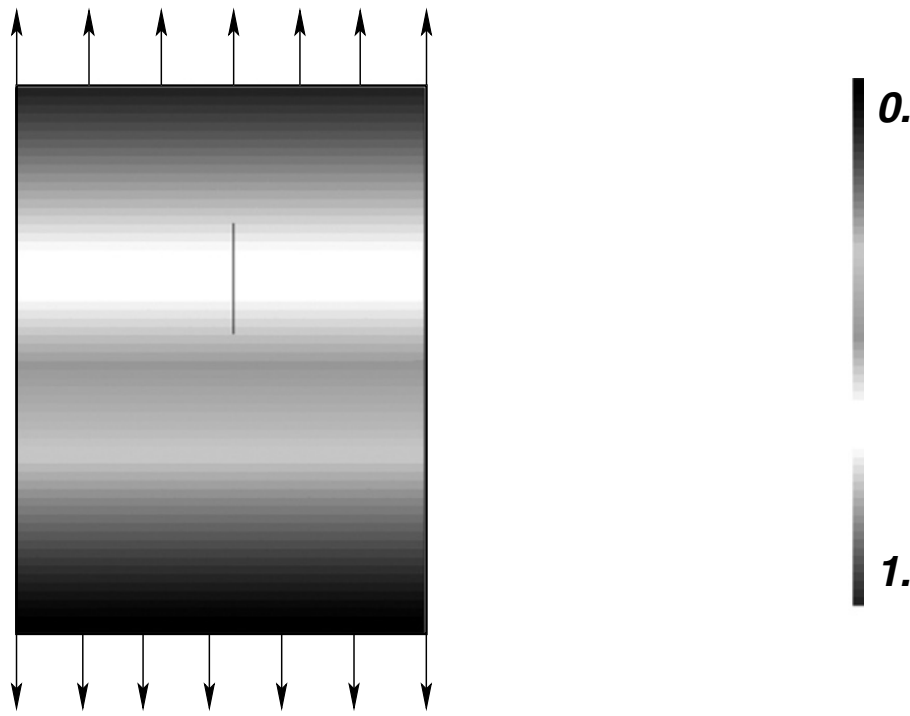

Figure 13.2. Example of a loading that does not enable the identification of planar cracks. Gray levels are isovalues of the potential $u$

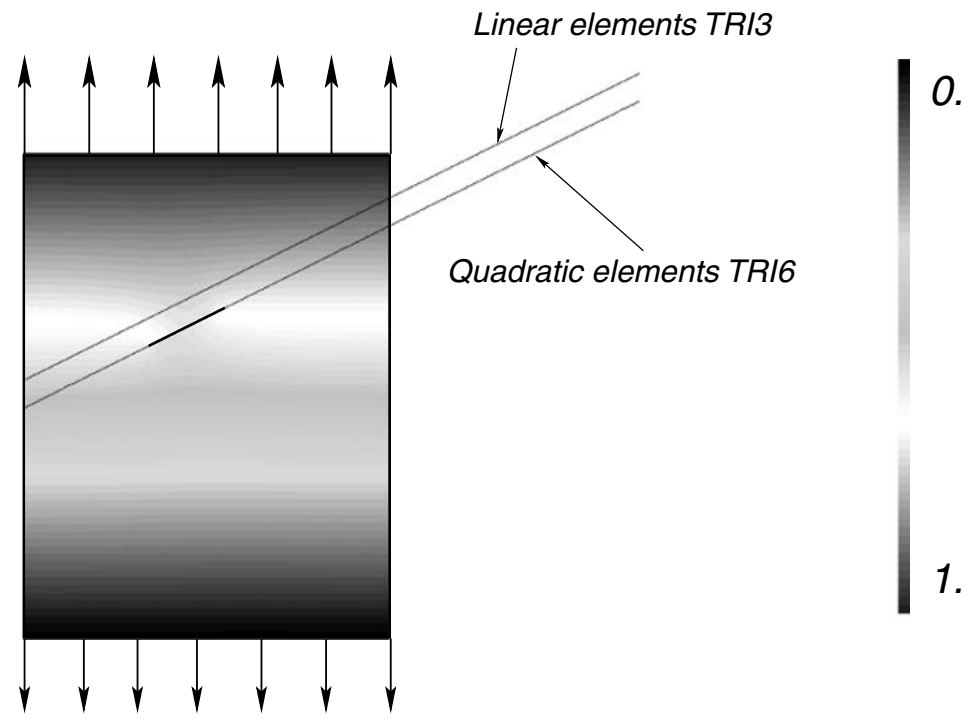

Figure 13.3. Identification example of the position of the plane of the crack for reconstructions using linear or quadratic finite elements for the representation of the temperature $T$ and the auxiliary function $v$ 


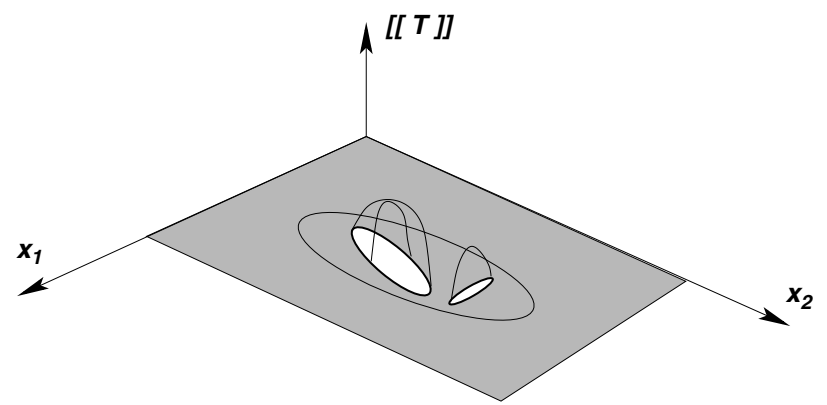

Figure 13.4. Extension of the temperature discontinuity $\llbracket T \rrbracket$ with a zero field in a rectangle $R$ containing the intersection of plane $\Pi$ with body $\Omega$

By extending the temperature jumps $\llbracket T \rrbracket$ with zero outside the cracks in the rectangle $R=[0, L] \times[0, H]$, which contains the intersection of plane $\Pi$ with body $\Omega$, and by using auxiliary fictitious functions $w_{p q}^{c c}, w_{p q}^{c s}, w_{p q}^{s c}$, which are a combination of sine and cosine functions, defined as:

$$
\begin{aligned}
w_{p q}^{s s}\left(X_{1}, X_{2}, X_{3}\right) & =\frac{1}{\sqrt{\lambda_{p q}}} \sin \left(\rho \pi \frac{X_{1}}{L}\right) \sin \left(\rho \pi \frac{X_{2}}{H}\right) \sinh \left(\sqrt{\lambda_{p q}} X_{3}\right) \\
\lambda_{p q} & =\pi^{2}\left(\frac{p^{2}}{L^{2}}+\frac{q^{2}}{H^{2}}\right)
\end{aligned}
$$

we obtain the following expression of the reciprocity gap:

$$
\mathcal{R}\left(w_{p q}^{s s}\right)=\int_{R} \llbracket T \rrbracket \sin \left(\rho \pi \frac{X_{1}}{L}\right) \sin \left(\rho \pi \frac{X_{2}}{H}\right) d X_{1} d X_{2}
$$

This expression enables us to recover the Fourier expansion of the temperature discontinuity $\llbracket T \rrbracket$.

Similar results have equally been obtained in linear isotropic elasticity (1) for the Lamé operator in [AND 92] and (2) for the Helmholtz operator in [BEN 05]. The result of the reconstruction using the Fourier expansion is shown in Figure 13.5.

\subsection{Crack identification in thermoelasticity using displacement measurements}

Let us consider a heterogeneous, isotropic and linear elastic body subjected only to thermal loading. The stress-strain relation is, in this case, expressed as:

$$
\boldsymbol{\sigma}=\boldsymbol{A}:\left(\varepsilon-\alpha T \boldsymbol{I}_{2}\right) \quad \boldsymbol{A} \frac{\nu E}{(1+\nu)(1-2 \nu)} \boldsymbol{I}_{2} \otimes \boldsymbol{I}_{2}+\frac{E}{1+\nu} \boldsymbol{I}_{4}
$$


where $\alpha$ is the thermal expansion coefficient, $E$ is the Young's modulus, $\nu$ is the Poisson coefficient and $T$ is the temperature increase with respect to the reference temperature of the material. $\boldsymbol{I}_{2}$ and $\boldsymbol{I}_{4}$ will denote the fourth- and second-order identity tensors, respectively.

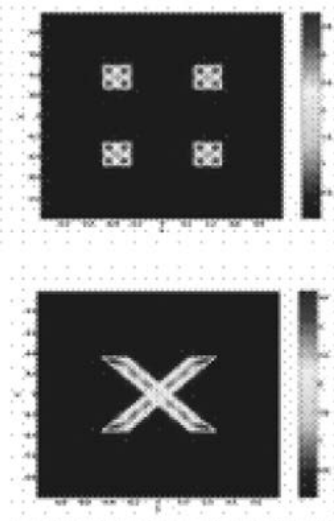

Exact
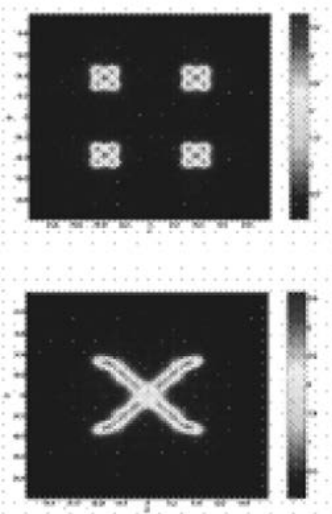

Reconstructed

Figure 13.5. Multiple cracks and non-convex cracks: temperature discontinuities, original fields and their reconstructions [BEN 05]

An interesting test case is that of imposed, but not measured, thermal loading on a traction-free solid. The measurement will imply only the displacement field $\boldsymbol{u}^{r}$ on the exterior surface.

In the case of small strain and neglecting inertial forces, the thermomechanical balance equations and the transient heat equation are expressed by the following expressions:

$$
\text { Thermal evolution }
$$

in $\Omega \backslash \Gamma \quad \rho c \dot{T}-\operatorname{div} k \nabla T=s$

$$
\text { on } \Gamma \quad k \nabla T \cdot \boldsymbol{N}=r_{T}(\llbracket T \rrbracket)
$$$$
\text { on } \partial \Omega \quad k \nabla T \cdot \boldsymbol{n}=\Phi
$$

on $\partial \Omega$

in $\Omega \backslash \Gamma \quad T=T^{0}$
Thermoelastic balance

$\operatorname{div} \sigma=0$

$\boldsymbol{\sigma} \cdot \boldsymbol{n}=r(\llbracket u \rrbracket)$

$\boldsymbol{\sigma} \cdot \boldsymbol{n}=\mathbf{0}$

$\boldsymbol{u}=\boldsymbol{u}^{D}$

where $s$ is the heat source, $\Phi$ is the surface flux, $T_{0}$ is the initial temperature, $r_{T}$ is the thermal resistance and $r$ is the contact stiffness of the cracks. All these parameters are considered to be unknown. 
The reciprocity gap in terms of mechanical fields is defined for each time instant $t \in(0, D)$ in the now usual sense for the displacements and traction fields by the formula:

$$
\mathcal{R}_{t}(v)=-\int_{\partial \Omega} \boldsymbol{u}^{r} \cdot(\boldsymbol{A}: \boldsymbol{\varepsilon}((v)) \cdot \boldsymbol{n}) d s
$$

where the traction-free condition at the exterior surface of the body has already been taken into account.

For auxiliary displacement fields $\boldsymbol{v}$, with the additional properties of assuring the balance offorces and zero divergence, the interpretation of the reciprocity gap is given by the following equation:

$$
\mathcal{R}_{t}(v)=\int_{\Gamma} \llbracket u \rrbracket \cdot(\boldsymbol{A}: \boldsymbol{\varepsilon}(\boldsymbol{v}) \cdot \boldsymbol{N}) d s
$$

for all auxiliary displacement fields of the set:

$$
\mathcal{V}_{\mathrm{div}}=\left\{\boldsymbol{v} \mid \int_{\Omega} \varepsilon(\boldsymbol{v}): \boldsymbol{A}: \boldsymbol{\varepsilon}(\boldsymbol{w})=\int_{\partial \Omega}(\boldsymbol{A}: \boldsymbol{\varepsilon}(\boldsymbol{v})) \cdot \boldsymbol{n} \forall \boldsymbol{w} \text { with } \operatorname{div} \boldsymbol{w}=0\right\}
$$

The zero divergence property of the auxiliary fields partly erases the coupling with the thermal problem. As a result, we can exploit only the displacement measurements at the surface of the body and neglect the information about its thermal state.

Let us define the following auxiliary displacement fields with zero divergence:

$$
\begin{aligned}
& \boldsymbol{v}_{i}(\boldsymbol{x})=2\left(3 x_{i} \boldsymbol{e}_{i}-\boldsymbol{x}\right) \quad i=1,3 \\
& \boldsymbol{w}(\boldsymbol{x})=2\left(x_{3} x_{2} \boldsymbol{e}_{1}+x_{1} x_{3} \boldsymbol{e}_{2}+x_{2} x_{1} \boldsymbol{e}_{3}\right)
\end{aligned}
$$

If the cracks are included in the plane $\Pi$ of normal $\boldsymbol{N}$, we obtain the following expressions for the reciprocity gap tensor:

$$
\begin{aligned}
\hat{\boldsymbol{R}} & =\operatorname{dev} \boldsymbol{R}=\boldsymbol{R}-\frac{1}{3}(\operatorname{tr} \boldsymbol{R}) \boldsymbol{I}_{2} \\
\boldsymbol{R} & =\frac{1}{2}(\boldsymbol{N} \otimes \boldsymbol{U}+\boldsymbol{U} \otimes \boldsymbol{N}) \\
\boldsymbol{U} & =\int_{\Gamma} \llbracket \boldsymbol{u} \rrbracket d s
\end{aligned}
$$


The components of the tensor are related to the reciprocity gap as expressed in the following relations:

- If $i \neq j$, then for $k$ different from indices $i$ and $j$, we have:

$$
\hat{R}_{i j}=\frac{1}{8 \mu} \mathcal{R}_{t}\left(\boldsymbol{w}_{, k}\right) \quad k \neq j k \neq j
$$

- If $i=j$, then:

$$
\hat{R}_{i i}=\frac{1}{12 \mu} \mathcal{R}_{t}\left(\boldsymbol{v}_{i}\right) \quad \text { (without summing) }
$$

The main question is how to retrieve the normal unit vector $N$ and the vector field of the displacement dicontinuity $\boldsymbol{U}$ from the knowledge of $\hat{\boldsymbol{R}}$.

Let us consider the eigenvalues $\left(\lambda_{1}, \lambda_{2}, \lambda_{3}\right)$ of $\hat{\boldsymbol{R}}$, in decreasing order of corresponding unit eigenvectors $\left(\boldsymbol{g}_{1}, \boldsymbol{g}_{2}, \boldsymbol{g}_{3}\right)$. The vectors $\boldsymbol{N}$ and $\boldsymbol{U}$ are in one of the two cases: they are either (1) colinear or (2) not colinear.

If $\boldsymbol{N}$ and $\boldsymbol{U}$ are colinear vectors, then we have:

$$
U=U N
$$

and as a necessary condition, we further have $\boldsymbol{N}=\boldsymbol{g}_{1}$. Because $\boldsymbol{R}=U \boldsymbol{N} \otimes \boldsymbol{N}$, the eigenvalues of $\hat{\boldsymbol{R}}$ are related to $\boldsymbol{U}$ by the following relations:

$$
\lambda_{1}=\frac{2}{3} U \quad \lambda_{2}=\lambda_{3}=-\frac{1}{3} U
$$

if $U>0$.

If $\boldsymbol{N}$ and $\boldsymbol{U}$ are not colinear vectors, let us note:

$$
\boldsymbol{U}=U \boldsymbol{d}_{u}
$$

Then, we can easily prove that $\boldsymbol{u} \times \boldsymbol{N}$ is colinear with $\boldsymbol{g}_{3}$. As a result, we obtain:

$$
\boldsymbol{N}=n_{1} \boldsymbol{g}_{1}+n_{3} \boldsymbol{g}_{3} \quad \boldsymbol{d}_{u}=n_{1} \boldsymbol{g}_{1}-n_{3} \boldsymbol{g}_{3}
$$

A complete discussion of the order of eigenvalues [AND 06] and the assumption that $U>0$ lead to the following relationship for directions 1 and 3 :

$$
n_{1}=\frac{\sqrt{\lambda_{1}-\lambda_{2}}}{\sqrt{\lambda_{1}-\lambda_{3}}} \quad n_{3}=\frac{\sqrt{\lambda_{2}-\lambda_{3}}}{\sqrt{\lambda_{1}-\lambda_{3}}}
$$


It is important to note that it is impossible to distinguish between the directions $\boldsymbol{N}$ and $\boldsymbol{U}$.

In a practical application, it must be considered that $N$ will stay constant over time while $\boldsymbol{u}$ varies, in general, with the load and therefore with time.

Finally, the amplitude of the displacement jump $U$ is given by:

$$
U=\left\|\int_{\Gamma} \llbracket \boldsymbol{u} \rrbracket d s\right\|=\sqrt{\lambda_{1}-\lambda_{3}}
$$

Once the normal is known (using the above formulas at least two different time instants), the following change of coordinates can be performed: the vector $\boldsymbol{E}_{3}$ is taken parallel to the normal to plane $\Pi$, which is now defined by $\boldsymbol{x} \cdot \boldsymbol{e}_{3}+C=0$.

We only need to identify constant $C$ locating the affine plane containing the cracks. This follows from the reciprocity gap computation for the auxiliary displacement field $\boldsymbol{h}$ :

$$
C=-\frac{1}{6 \mu \int_{\Gamma} \llbracket u_{x} \rrbracket d s} \mathcal{R}_{t}(\boldsymbol{h}) \quad \text { with } \quad \boldsymbol{h}=3\left(x_{3}^{2}-x_{2}^{2}\right) \boldsymbol{e}_{1}
$$

\subsection{Conclusions and perspectives}

The reciprocity gap technique has a great advantage: that is its simplicity. A number of identification problems have been solved from a theoretical or numerical point of view. The identification of planar cracks for different families of auxiliary fields has been discussed in the following cases:

- scalar diffusion equation, that is steady-state heat equation or electric conductivity in [AND 92, AND 99, AND 96];

- transient heat equation in [BEN 01];

- transient acoustics (wave propagation with a scalar acoustic potential) in [BUI 99]:

- elastostatics (diffusion equation with vector potential) in [BAN 97, BAN 99]

- elastodynamics (wave propagation with vector potential) in [BUI 04, BUI 05];

- small strain coupled thermoelasticity in [AND 06];

- damped elastodynamics with Zehner-type viscoelasticity (Helmholtz equation) in [BUI 10];

- acoustic dispersion (Helmholtz equation with a scalar potential) [COL 05, BEN 05]; 
The disadvantage of the method, as indeed for a number of inverse problems, is the necessity of knowing the measured fields over the complete outer boundary of the body. The search for suitable ways to overcome this difficulty remains an open problem for the years to come.

\subsection{Bibliography}

[AND 92] ANdrieux S., Ben AbDa A., "Identification des fissures planes par une donnée de bord unique: un procédé direct de localisation et d'identification", Comptes Rendus de l'Académie des Sciences (Série I), vol. 315, pp. 1323-1328, 1992.

[AND 96] ANDRIEUX S., BEN ABDA A., "Identification of planar cracks by complete overdetermined data: inversion formulae", Inverse Problems, vol. 12, no. 5, pp. 553-563, 1996.

[AND 99] ANdrieux S., Ben Abda A., Bui H.D., "Reciprocity principle and crack identification", Inverse Problems, vol. 15, no. 1, pp. 59-65, 1999.

[AND 06] ANDRIEUX S., Bui H.D., "Écart à la réciprocité et identification de fissures en thermo-élasticité isotrope transitoire", Comptes Rendus de l'Académie des Sciences (Série I), vol. 334, no. 4, pp. 225-229, 2006.

[BAN 97] Bannour T., Ben ABda A., JaOuA M., "A semi-explicit algorithm for the reconstruction of 3D planar cracks", Inverse Problems, vol. 13, no. 4, pp. 899-917, 1997.

[BAN 99] Bannour T., Ben Abda A., JaOua M., "Identification of 2D cracks by elastic boundary measurements", Inverse Problems, vol. 15, no. 1, pp. 67-77, 1999.

[BEN 01] BeN ABDA A., Bui H.D., "Reciprocity principles and crack identification in transient thermal problems", Inverse and Ill-Posed Problems, vol. 9, no. 1, pp. 1-6, 2001.

[BEN 05] Ben Abda A., Delbary F., Haddart H., "On the use of the reciprocity-gap functional in inverse scattering from planar cracks", Mathematical Models and Methods in Applied Sciences, vol. 15, no. 10, pp. 1553-1574, 2005.

[BUI 99] Bui H.D., Constantinescu A., Maigre H., "Diffraction acoustique inverse de fissure plane: solution explicite pour un solide borné", Comptes Rendus de l'Académie des Sciences (série II), vol. 327, pp. 971-976, 1999.

[BUi 04] Bui H., Constantinescu A., Maigre H., "Numerical identification of planar cracks in elastodynamics using the instantaneous reciprocity gap", Inverse Problems, vol. 20, pp. 993-1001, 2004.

[BUi 05] Bui H.D., Constantinescu A., Maigre H., "An exact inversion formula for determining a planar fault from boundary measurements", Inverse and Ill-Posed Problems, vol. 13 , no. 6 , pp. 553-565, 2005.

[BUi 10] Bui H.D., Chaillat S., Constantinescu A., Grasso, E., "Identification of a planar crack in Zener type viscoelasticity", Annals of Solid and Structural Mechanics, vol. 1, pp. 3-8, 2010,

[COL 05] Colton D., Haddar H., "An application of the reciprocity gap functional to inverse scattering theory”, Inverse Problems, vol. 21, no. 1, pp. 383-398, 2005. 